\title{
Optimization of Storage Conditions of the Medicinal Herb Ilex asprella against the Sterigmatocystin Producer Aspergillus versicolor Using Response Surface Methodology
}

\author{
Xiaofang $\mathrm{Lu}^{1,2,3}$, Chaoquan Luo ${ }^{1,2,3}$, Jianyong Xing ${ }^{4}$, Zhengzhou Han ${ }^{4}$, Tong $\mathrm{Li}^{5}$, Weiwei $\mathrm{Wu}^{5}$, \\ Hui $\mathrm{Xu}{ }^{1,2,3, *}$, Ruoting Zhan ${ }^{1,2,3}$ and Weiwen Chen ${ }^{1,2,3}$ \\ 1 Research Center of Chinese Herbal Resource Science and Engineering, Guangzhou University of Chinese \\ Medicine, Guangzhou 510006, China; 1xf8717@gmail.com (X.L.); 512684691@qq.com (C.L.); \\ ruotingzhan@vip.163.com (R.Z.); chenww@gzucm.edu.cn (W.C.) \\ 2 Ministry of Education Key Laboratory of Chinese Medicinal Resource from Lingnan, \\ Guangzhou 510006, China \\ 3 Joint Laboratory of National Engineering Research Center for the Pharmaceutics of Traditional Chinese \\ Medicines, Guangzhou 510006, China \\ 4 China Resources Sanjiu Medical \& Pharmaceutical Co, Ltd., Shenzhen 518110, China; \\ xingjianyong@999.com.cn (J.X.); gulf@999.com.cn (Z.H.) \\ 5 School Materia Medica, Guangzhou University of Chinese Medicine, Guangzhou 510006, China; \\ litong9801@outlook.com (T.L.); wuweiwei717@outlook.com (W.W.) \\ * Correspondence: zyfxsherry@gzucm.edu.cn; Tel./Fax: +86-20-3935-8331
}

Received: 22 October 2018; Accepted: 20 November 2018; Published: 27 November 2018

\begin{abstract}
The root of Ilex asprella is a commonly used herb in Southern China, and also constitutes the main raw material of Canton herbal tea. I. asprella is readily contaminated by mildew because of rich nutrients. Aspergillus versicolor producing sterigmatocystin is one of the most common molds that contaminate foodstuffs and medicinal herbs. Previous study on the evaluation of fungal contamination on samples of $I$. asprella revealed that $A$. versicolor was the dominant contaminant. In this study, experiments based on response surface methodology combined with central composite design were carried out to determine the optimal storage conditions of I. asprella to minimize the contamination of sterigmatocystin. The herb, manually innoculated with A. versicolor, was stored under different temperatures $\left(20-40{ }^{\circ} \mathrm{C}\right)$ and humidity $(80-95 \%)$ for seven days. The effects of temperature and humidity were evaluated using total saponin, polysaccharide and the sterigmatocystin levels as quality indexes. The results showed that $A$. versicolor grew quickly and produced large amounts of sterigmatocystin on I. asprella, at humidity ranging from $85 \%$ to $90 \%$ and temperatures above $26^{\circ} \mathrm{C}$. Meanwhile, total saponin and polysaccharide amounts were reduced significantly. These findings suggested that $I$. asprella samples should be stored in an environment with humidity and temperature below $85 \%$ and $26^{\circ} \mathrm{C}$, respectively, to reduce $A$. versicolor growth and sterigmatocystin production.
\end{abstract}

Keywords: Ilex asprella; Aspergillus versicolor; sterigmatocystin; response surface methodology; storage conditions; temperature; humidity

Key Contribution: The optimal storage conditions of the medicinal herb I. asprella to protect against sterigmatocystin producers were determined based on response surface methodology combined with central composite design. Samples stored at a humidity and temperature below $85 \%$ and $26{ }^{\circ} \mathrm{C}$ respectively, could significantly reduce the accumulation of sterigmatocystin (ST) while maintaining the amounts of active ingredients. 


\section{Introduction}

Toxigenic fungi are widely distributed in nature, and not only pollute crops, but also contaminate medicinal plants [1,2]. Halt et al. [3] performed a pollution survey of 62 medicinal plants in Croatia, of which $18 \%$ were contaminated by toxigenic fungi.

Aspergillus versicolor producing sterigmatocystin (ST) is one of the most common mildews that contaminate foodstuffs and medicinal herbs. ST has obvious carcinogenicity and mutagenicity for animals and humans, with toxicity only inferior to aflatoxin [4]. In addition, ST is an intermediate product of aflatoxin biosynthesis [5]. The International Cancer Research Institute (IARC) classifies ST as a Class 2B carcinogen. ST is also produced by different Aspergillus species as well as other species such as Bipolaris, Chaetomium and Emiricella [6]. Vesonder et al. reported that multiple dairy cattle had bloody flux, which reduced milk production, with some animals dying; this was confirmed to be caused by ST in animal feed [7]. In the YinNa areas of Northwest China, a flock of sheep was reported to suffer from the so-called "yellow" disease for a long time; examination revealed that the animals were fed mildew contaminated grass in winter, with ST content as high as $6.5 \mathrm{mg} / \mathrm{kg}$ [8]. Recently, Zheng et al. [9] assessed 365 samples of medicinal herbs collected in Guangzhou, China, and ST was detected in one fourth of them.

Ilex asprella is one of the most popular medicinal herbs in South China. The root of I. asprella is rich in triterpenoid saponins, and has various pharmacological effects; it has been used to treat wind-heat cold, chronic pharyngitis and sore throat [10]. As a "drug homologous food", it is also included in many herbal teas. I. asprella is highly susceptible to mildew, which might result from the extra large amount of polysaccharides it contains, with a content of up to $10 \%$ [11]. In our previous study, fungi on the surface of 22 commercial I. asprella samples were isolated, purified and identified by ITS sequencing as reported by Bellemain et al. [12]. In total, 29 fungal strains were isolated, including six proven to be ST producers as determined by LC-MS/MS. The above findings implied that the contamination risk of ST producers for I. asprella can be quite high and should not be overlooked.

To minimize fungal infection and mycotoxin accumulation, it is necessary to store medicinal herbs and foodstuffs in appropriate conditions. Based on previous reports, temperature and humidity are two major environmental factors $[13,14]$. Therefore, in the present study, manually inoculated I. asprella samples were stored under various temperature and humidity conditions, designed by response surface methodology combined with central composite design (RSM-CCD), and the sample quality was evaluated to provide clues for optimal storage conditions against ST producing fungi. The standard strain $A$. versicolor was selected as a representative strain to inoculate samples. Polysaccharides, total saponins and sterigmatocystin (ST) were used as quality indexes.

\section{Results and Discussion}

\subsection{Growth of A. versicolor after Manual Inoculation}

After inoculation with $A$. versicolor suspension, I. asprella samples were incubated under specific temperature and humidity conditions for seven days. A. versicolor growth varied greatly among different test groups, and luxuriant growth was observed at the temperature of $30{ }^{\circ} \mathrm{C}$ and relative humidity of $88 \%$. Typical samples observed under the sunlight are shown in Figure 1. 

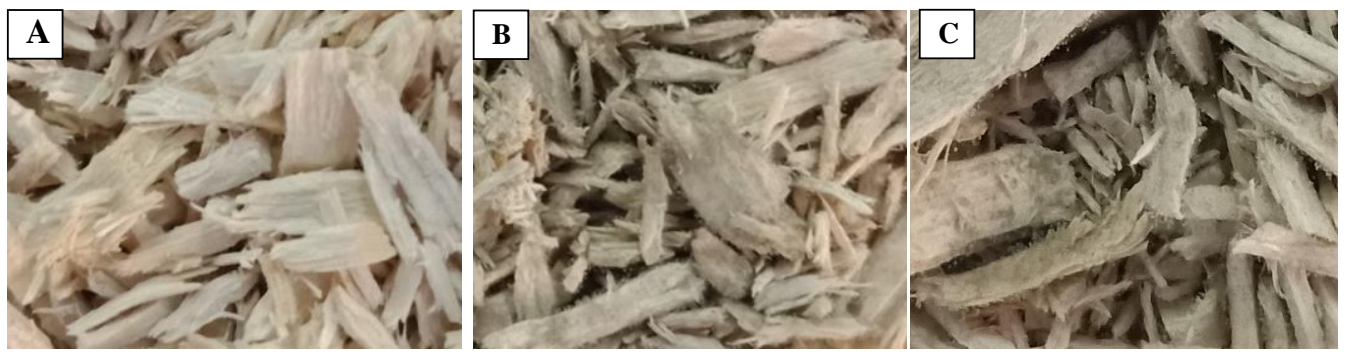

Figure 1. Typical samples observed under the sunlight. (A) negative control; (B) inoculated sample after incubation for seven days at $23{ }^{\circ} \mathrm{C}$ and $82 \%$ humidity; (C) inoculated sample after incubation for seven days at $30^{\circ} \mathrm{C}$ and $88 \%$ humidity.

\subsection{Sterigmatocystin Content in I. asprella}

After incubation for seven days, ST levels in I. asprella samples were determined by a HPLC-MS/MS method developed previously [9]. Typical SRM chromatograms are shown in Figure 2. ST contents ranged from 8.4 to $19.6 \mu \mathrm{g} / \mathrm{kg}$ (Table 1).

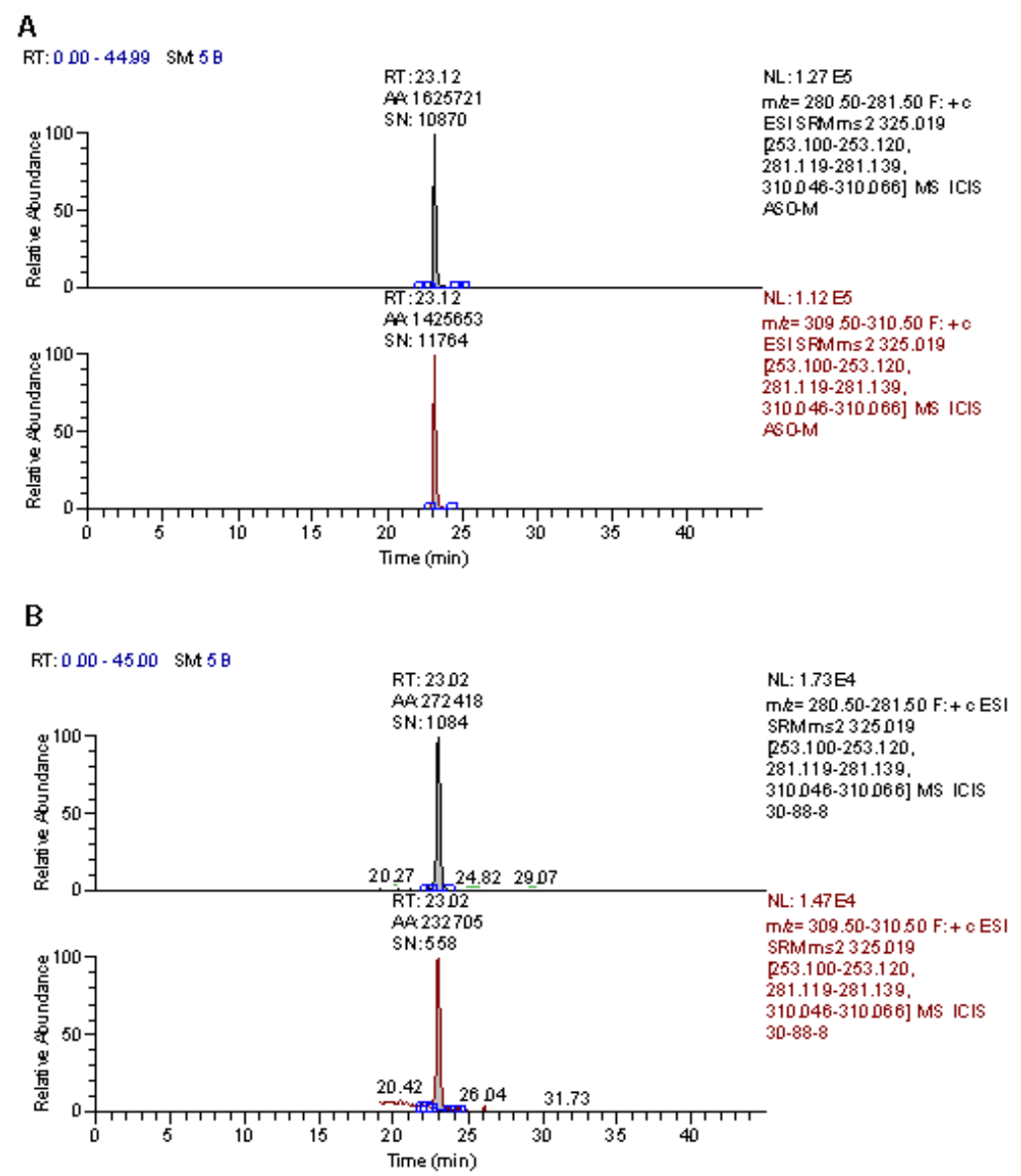

Figure 2. Cont. 


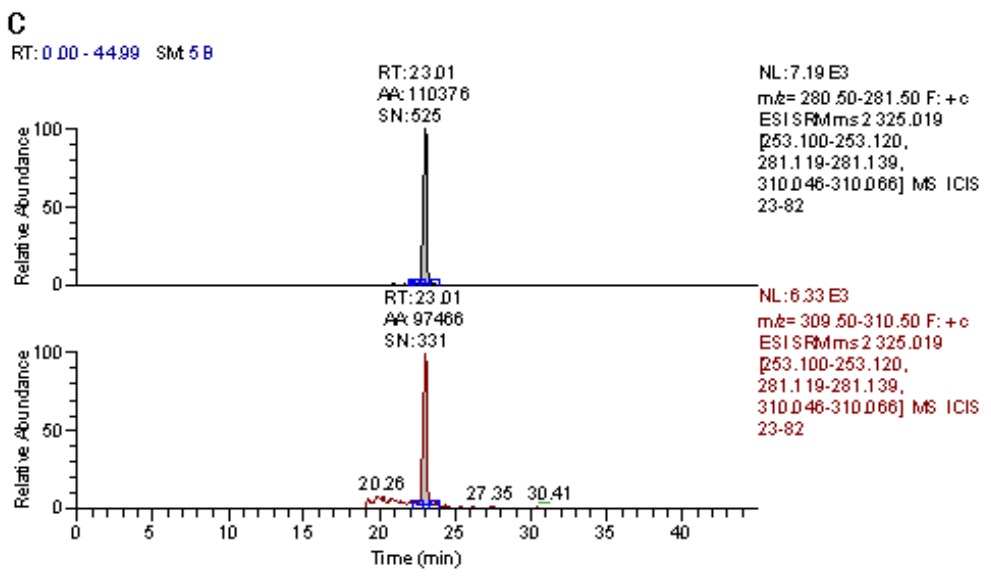

Figure 2. Typical SRM chromatograms of sterigmatocystin (ST) containing I. asprella samples. (A) ST standard; (B) inoculated sample after incubation for seven days at $30{ }^{\circ} \mathrm{C}$ and $88 \%$ humidity; (C) inoculated sample after incubation for seven days at $23{ }^{\circ} \mathrm{C}$ and $82 \%$ humidity. Top, quantitative ion; bottom, qualitative ion.

Table 1. Outline of experimental design and the responses observed in I. asprella samples.

\begin{tabular}{cccccc}
\hline Test & Temp. & Hum. & $\begin{array}{c}\text { ST } \\
(\boldsymbol{\mu g} / \mathbf{k g})\end{array}$ & $\begin{array}{c}\text { Total Saponins } \\
(\mathbf{m g} / \mathbf{g})\end{array}$ & $\begin{array}{c}\text { Polysaccharides } \\
(\mathbf{\%})\end{array}$ \\
\hline 1 & 23 & 82 & 13.0 & 6.48 & 9.93 \\
2 & 37 & 82 & 8.8 & 4.47 & 9.45 \\
3 & 23 & 93 & 11.2 & 4.67 & 7.48 \\
4 & 37 & 93 & 11.6 & 3.86 & 4.39 \\
5 & 20 & 88 & 10.6 & 7.07 & 9.73 \\
6 & 40 & 88 & 10.6 & 4.12 & 11.38 \\
7 & 30 & 80 & 8.4 & 6.21 & 9.4 \\
8 & 30 & 95 & 12.2 & 2.25 & 6.28 \\
9 & 30 & 88 & 18.0 & 3.52 & 5.03 \\
10 & 30 & 88 & 19.6 & 1.83 & 4.41 \\
11 & 30 & 88 & 18.8 & 3.1 & 4.66 \\
12 & 30 & 88 & 17.2 & 2.5 & 4.28 \\
13 & 30 & 88 & 16.8 & 2.17 & 4.72 \\
\hline
\end{tabular}

\subsection{Polysaccharide and Total Saponin Contents}

To quantify the polysaccharide content of I. asprella, the phenol-sulfuric acid method was used. The calibration curve generated with glucose standard was linear within the range of $5-50 \mu \mathrm{g} / \mathrm{mL}$, with a correlation coefficient of 0.99 . The average polysaccharide content of $I$. asprella was $12.71 \%$. After incubation with $A$. versicolor for seven days, the polysaccharide content was reduced remarkably (Table 1), especially for samples stored at $30^{\circ} \mathrm{C}$ and $88 \%$ humidity. Furthermore, under these conditions, A. versicolor grew well, with very high ST concentrations. To assess total saponin amounts in I. asprella, colorimetry detecting vanillin-glacial acetic acid was applied. The calibration curve generated with ursolic acid was linear within the range of $25-400 \mu \mathrm{g} / \mathrm{mL}$, with a correlation coefficient of 0.99 . The average content of total saponins was $10.83 \mathrm{mg} / \mathrm{g}$. Similar to polysaccharide, total saponin amounts were reduced remarkably after incubation, as shown in Table 1.

\subsection{Model Fitting and Statistical Analysis}

The experiments showed that all variables examined in this study had effects on ST, total saponin amounts and polysaccharide production. Therefore, the effects of two variables, including temperature and humidity on responses (levels of ST, total saponins and polysaccharides) were examined using central composite design (CCD). Table 1 depicts the complete design matrix as well as response values. Multiple regression analysis provided predicted responses ( $\mathrm{Y}$ values) for ST, total saponins and polysaccharides, via second-order polynomial Equations (1)-(3) as follows: 
ST: $Y=-0.057317 \times T^{2}-0.11841 \times H^{2}+0.030847 \times T \times H+0.66258 \times T+19.89322 \times H-865.79897$

Total saponins: $Y=0.028156 \times T^{2}+0.023946 \times H^{2}+0.00739582 \times T \times H-2.46118 \times T-4.59722 \times H+250.82892$

Polysaccharides: $Y=0.049183 \times T^{2}+0.036444 \times H^{2}-0.015132 \times T \times H-1.64457 \times T-6.19986 \times H+313.28930$

where $Y$ is the predicted response (levels of ST, total saponins and polysaccharides, respectively); $T$ and $H$ represent two independent variables, temperature and humidity, respectively.

Table 2 summarizes the data for analysis of variance by Fisher's $F$ test, the goodness-of-fit and the adequacy of all models. For ST, a quadratic regression model with high significance was found with high $F$ and extremely low $p$ - $(p=0.0065)$ values, suggesting the combined impacts of different independent variables markedly contributed to response maximization [15]. Meanwhile, there was lack of fit $(p=0.3397)$ confirming that these data fit well with this model. A determination coefficient $\left(R^{2}\right)$ of 0.8963 indicated that a variation of $89.63 \%$ for ST production was due to independent parameters in samples. Equation (1) equally had an adjusted correlation coefficient $\left(R^{2}{ }_{\text {adj }}\right)$ of 0.8099 , which satisfactorily confirmed the model's significance [16]. The higher the $R^{2}$ adj value, the more actual and predicted values are correlated [17]. For Equation (2), $(p<0.05)$ and an F-value of 11.55 indicated a useful model. An $R^{2}$ of 0.9060 suggested about $9.4 \%$ of the overall variance could not be due to the response. $R^{2}$ adj was 0.8120 , suggesting a reliable mathematical model, which was suitable for determining total saponin amounts. For Equation (3), $p<0.05$ and an $F$-value of 9.51 indicated a useful model. $R^{2}$ of 0.8879 was derived, for an $R^{2}$ adj of 0.7945 , indicating the mathematical model had good reliability, and could be applied to assess polysaccharide amounts. Moreover, $p$ values of humidity were higher than those of temperature in all three models, indicating that the former demonstrated more significant influence on I. asprella quality than the latter.

Table 2. Response surface quadratic models given by ANOVA for assessing target mycotoxin, total saponin amounts and polysaccharide production.

\begin{tabular}{cccccc}
\hline Source & Sum of Squares & df & Mean Square & F-Value & $p$-Value \\
\hline ST & & & & & \\
Model & 74.2 & 5 & 14.84 & 10.37 & 0.0065 \\
Temp & 2.22 & 1 & 2.22 & 1.55 & 0.2597 \\
Hum & 1.85 & 1 & 1.85 & 1.29 & 0.2993 \\
TH & 5.68 & 1 & 5.68 & 3.97 & 0.0935 \\
$\mathrm{~T}^{2}$ & 25.35 & 1 & 25.35 & 17.72 & 0.0056 \\
$\mathrm{H}^{2}$ & 62.73 & 1 & 62.73 & 43.84 & 0.0006 \\
Residual & 8.59 & 6 & 1.43 & & \\
Lack of fit & 5.39 & 3 & 1.80 & 1.68 & 0.3397 \\
Pure Error & 3.20 & 3 & 1.07 & & \\
Cor Total & 185.76 & 12 & & & \\
\hline Total saponins & & & & & \\
Model & 29.39 & 5 & 5.88 & 11.55 & 0.0049 \\
Temp & 6.13 & 1 & 6.13 & 12.05 & 0.0133 \\
Hum & 7.97 & 1 & 7.97 & 15.67 & 0.0075 \\
TH & 0.33 & 1 & 0.33 & 0.64 & 0.4542 \\
$\mathrm{~T}^{2}$ & 12.98 & 1 & 12.98 & 25.51 & 0.0023 \\
H ${ }^{2}$ & 3.10 & 1 & 3.10 & 6.09 & 0.0486 \\
Residual & 3.05 & 6 & 0.51 & & \\
Lack of fit & 2.17 & 3 & 0.72 & 2.48 & 0.2379 \\
Pure Error & 0.88 & 3 & 0.29 & & \\
Cor Total & 35.57 & 12 & & & \\
\hline Polysaccharides & & & & & \\
Model & 63.44 & 5 & $12 . .69$ & 9.51 & 0.0081 \\
Temp & 0.12 & 1 & 0.12 & 0.092 & 0.7722 \\
Hum & 17.80 & 1 & 17.80 & 13.34 & 0.0107 \\
TH & 1.36 & 1 & 1.36 & 1.02 & 0.3512 \\
$\mathrm{~T}^{2}$ & 39.61 & 1 & 39.61 & 29.68 & 0.0016 \\
H ${ }^{2}$ & 7.18 & 1 & 7.18 & 5.38 & 0.0595 \\
Residual & 8.01 & 6 & 1.33 & & \\
Lack of fit & 7.81 & 3 & 2.60 & 40.08 & 0.0064 \\
Pure Error & 0.19 & 3 & 0.065 & & \\
Cor Total & 83.21 & 12 & & & \\
\hline & & & & & \\
\hline
\end{tabular}




\subsection{Response Surface Analysis}

The effects of the independent parameters, temperature and humidity, as well as their interactive impacts on the amounts of ST, total saponins and polysaccharides were depicted by 2-dimensional contour plots and 3-dimensional response surface plots, respectively, employing RSM. Regression models in Equations (1)-(3) helped predict responses, deriving ultimate values for ST, total saponins and polysaccharide levels $[18,19]$.

As shown in Figure 3, temperatures and humidity levels ranging from $26{ }^{\circ} \mathrm{C}$ to $34{ }^{\circ} \mathrm{C}$ and $85 \%$ to $90 \%$, respectively, significantly increased ST content, indicating that these conditions were optimal for A. versicolor to produce ST. Based on the data in Table 1, the temperature and humidity for the maximum production of ST were calculated to be $30{ }^{\circ} \mathrm{C}$ and $88 \%$, respectively. In comparison, increasing temperature ranging from $26^{\circ} \mathrm{C}$ to $37^{\circ} \mathrm{C}$ and relative humidity of $85-95 \%$ reduced the amounts of total saponins and polysaccharides remarkably (Figures 4 and 5). Therefore, to ensure optimal chemical composition for the herb as well as minimize ST-hazard risk, the recommended storage conditions would be humidity below $85 \%$ and temperature below $26^{\circ} \mathrm{C}$.
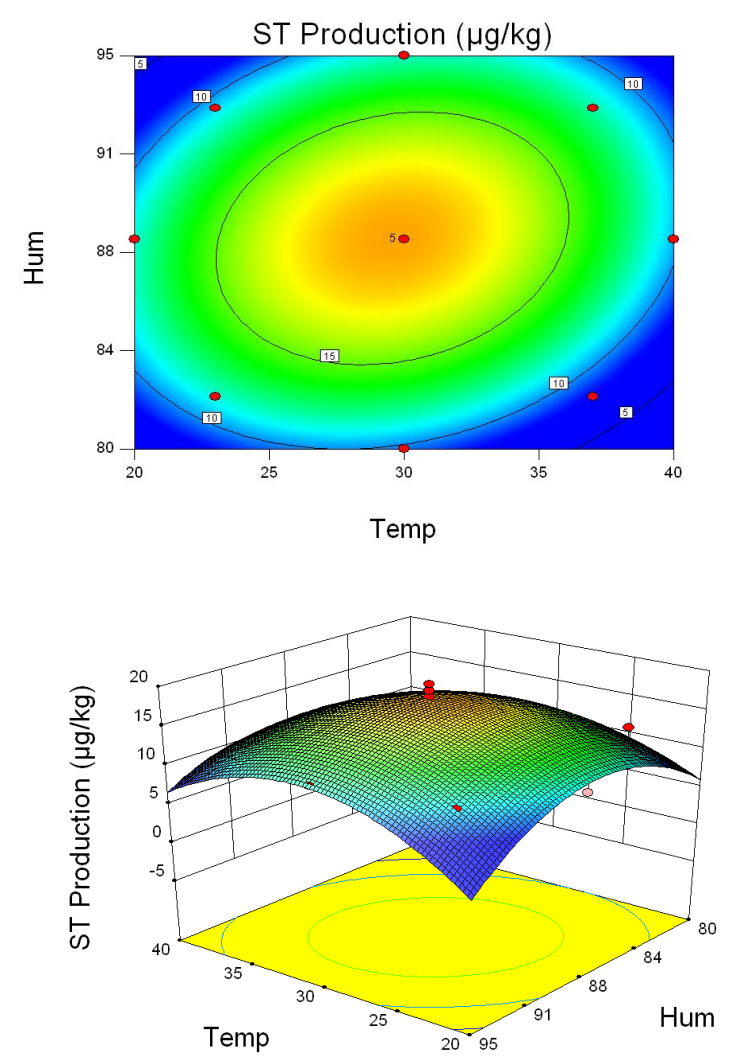

Figure 3. Three-dimensional response surface plots and corresponding contour plots of parameters for ST content in A. versicolor. The contact between temperature and humidity is shown. 

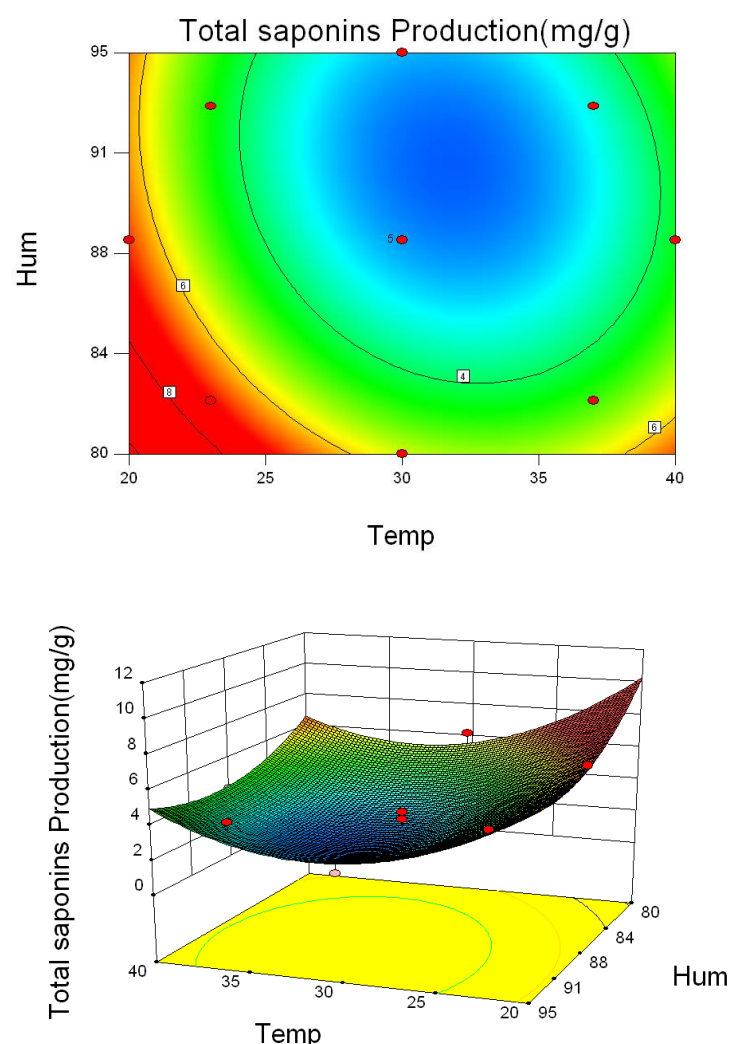

Figure 4. Three-dimensional response surface plots and corresponding contour plots of parameters for total saponin content in A. versicolor. The contact between temperature and humidity is shown.
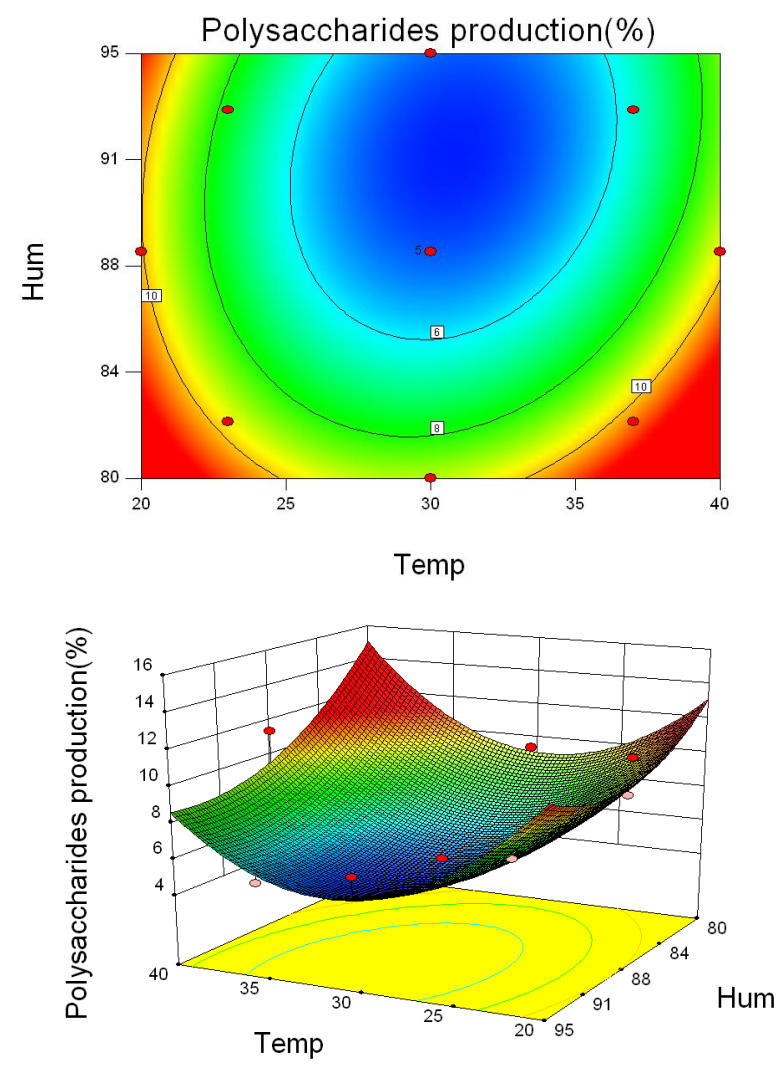

Figure 5. Three-dimensional response surface plots and corresponding contour plots of parameters for polysaccharide content in A. versicolor. The contact between temperature and humidity is shown. 


\section{Conclusions}

Stored I. asprella is an artificial ecosystem with qualitative and nutritive changes resulting from the interactions of physical, chemical and biological factors. Fungal damage and mycotoxin contamination constitute major concerns. Under satisfactory storage conditions, mycotoxin contamination can occur $[20,21]$. Elevated temperature and relative humidity represent critical parameters promoting mold growth and mycotoxin contamination during cereal, feed and herb storage [22]. I. asprella represents a commonly cultivated plant in tropical and subtropical areas, especially in Chinese Guangdong province, with a warm climate, heavy rainfall and high humidity. Therefore, I. asprella root might show elevated sensitivity to mildew during storage in these conditions.

Response surface methodology allows the determination of an optimal response based on a few sets of experiments, in which all factors vary within a chosen range. Conclusively, the proposed model has shown to be significantly important in statistical terms. This study showed that $A$. versicolor growth and toxicity were affected by environmental temperature and humidity. The effects of humidity on growth, mycotoxin production and the alteration of effective components were higher than those of temperature. Samples of I. asprella should be conserved under low temperature $26{ }^{\circ} \mathrm{C}$ and humidity $85 \%$, to reduce ST content, while maintaining the amounts of active ingredients. However, concerning the storage time, no conclusion could be drawn from this study. A long-term experiment with real samples might be necessary to figure out the answer. This study showed that it was scientific and feasible to use the RSD-CCD method to assess the conditions of herb storage, and provided new clues for controlling the pollution of medicinal plants by mycotoxin.

\section{Materials and Methods}

\subsection{Chemicals and Reagents}

Mycotoxin standard (ST) was purchased from Supelco Sigma Aldrich (St. Louis, MO, USA), dissolved in methanol $(1 \mu \mathrm{g} / \mathrm{mL})$ and stored at $-20{ }^{\circ} \mathrm{C}$ until analysis. A. versicolor strain As3.4413 was purchased from China General Microbiological Culture Collection Center (Beijing, China). Organic solvents, including methanol, acetonitrile and formic acid, were of HPLC grade and supplied by Merck (Merck KGaA, Germany). All the other organic reagents were of analytical grade and purchased from Tianjin Chemical Reagent (Tianjin, China). Pure water was obtained from a Milli-Q system (Millipore, Billerica, MA, USA).

\subsection{Plant Material Preparation and Pretreatment}

I. asprella roots were collected from fields, sliced and dried immediately. The resulting samples were stored in sealed bags at $-20^{\circ} \mathrm{C}$ until use. The samples $(10 \mathrm{~g}$ each) were sterilized by ultraviolet radiation for $1 \mathrm{~h}$. Then, $1.0 \mathrm{~mL}$ spore suspension of $A$. versicolor $\left(10^{6}\right.$ spores $\left./ \mathrm{mL}\right)$ was added accurately to the dish. According to the conditions described in Table 1, different storage conditions were simulated for seven days.

\subsection{Experimental Design}

In this study, RSM was performed to assess the associations of mycotoxin, total saponin and polysaccharide production. A two-factor, three-level design was developed by CCD to generate a second-order polynomial model. Then, two variables, namely temperature (A) and humidity (B), and their appropriate ranges were determined based on single-factor experiments.

The predicted and actual levels of the independent variables are presented in Table 3. 
Table 3. Experimental ranges and levels of the independent variables.

\begin{tabular}{|c|c|c|c|c|c|c|c|}
\hline \multirow{2}{*}{ Variables } & \multirow{2}{*}{ Symbols } & \multicolumn{5}{|c|}{ Range and Levels } & \multirow{2}{*}{ Remarks } \\
\hline & & -1 & -alpha & $\mathbf{0}$ & +alpha & +1 & \\
\hline \multirow{2}{*}{$\begin{array}{c}\text { Temperature } \\
\left({ }^{\circ} \mathrm{C}\right)\end{array}$} & \multirow{2}{*}{$\mathrm{T}$} & 22.93 & 20 & 30 & 40 & 37.03 & predicted \\
\hline & & 23 & 20 & 30 & 40 & 37 & actual \\
\hline \multirow{2}{*}{$\begin{array}{c}\text { Humidity } \\
\left({ }^{\circ} \mathrm{C}\right)\end{array}$} & \multirow{2}{*}{$\mathrm{H}$} & 82.2 & 80 & 87.5 & 95 & 92.8 & predicted \\
\hline & & 83 & 80 & 88 & 95 & 93 & actual \\
\hline
\end{tabular}

\subsection{Determination of Sterigmatocystin by HPLC-MS/MS}

Mycotoxin extraction from the plant was performed as previously described by our team [9]. Briefly, for extraction, $2.0 \mathrm{~g}$ of powder was diluted in $10 \mathrm{~mL}$ acetonitrile-water $(84 / 16, v / v)$. The sample was mixed for $60 \mathrm{~min}$ at maximum speed on a Vortex. The solution underwent centrifugation at $5000 \mathrm{rpm}$ for $5 \mathrm{~min}$ at room temperature. Afterward, the supernatant was decanted and filtered through a membrane with a $0.22 \mu \mathrm{m}$ Millipore filter. HPLC-MS/MS was used for ST purification and quantitation, using a Thermo Fisher Scientific Hypersil GOLD C 18 column $(2.1 \mathrm{~mm} \times 100 \mathrm{~mm}, 3 \mu \mathrm{m})$. The mobile phase, composed of methanol (A) and $4.0 \mathrm{mM}$ ammonium acetate $0.1 \%$ formic acid aqueous solution (B), was programmed as follows: gradient elution (0-5 min, $20 \% \mathrm{~A} ; 5-20 \mathrm{~min}, 20 \% \mathrm{~A}-80 \%$ A; $20-30 \mathrm{~min}, 80 \% \mathrm{~A}-100 \% \mathrm{~A} ; 30-35 \mathrm{~min}, 100 \% \mathrm{~A})$. The flow rate was $0.3 \mathrm{~mL} / \mathrm{min}$ and an injection volume of $10 \mu \mathrm{L}$ was adopted, with a column temperature at $30{ }^{\circ} \mathrm{C}$. Identification of the isolated mycotoxin was performed by HPLC-MS/MS in the HESI (+) mode and analytical HPLC by comparing retention times and MS data with analytical standards. For mass detection, the precursor $(m / z=325)$, quantitative $(m / z=281)$ and qualitative $(m / z=310)$ were used for ST analysis. ST detection of relevant mass spectrum parameters is shown in Table 4.

Table 4. HESI-MS/MS parameters of ST.

\begin{tabular}{cccc}
\hline Compound & $\begin{array}{c}\text { Collision energy } \\
(\mathbf{k v})\end{array}$ & $\begin{array}{c}\text { Quantitative Ion } \\
(\mathbf{m} / \mathbf{z})\end{array}$ & $\begin{array}{c}\text { Qualitative Ion } \\
(\mathbf{m} / \mathbf{z})\end{array}$ \\
\hline ST & $36 / 25$ & $325>281$ & $325>310$ \\
\hline
\end{tabular}

\subsection{Determination of Total Saponin Content}

The ursolic acid standard was diluted to a concentration $1 \mathrm{mg} / \mathrm{mL}$. For extraction, $1.0 \mathrm{~g}$ of powder was diluted in $20 \mathrm{~mL}$ of ethanol/water $(70: 30 \mathrm{v} / \mathrm{v})$, followed by ultrasonication for $30 \mathrm{~min}$. Filtration after cooling was performed using ethanol/water at constant volume of $50 \mathrm{~mL}$, and the solution underwent centrifugation at $8000 \mathrm{rpm}$ for $10 \mathrm{~min}$ at room temperature. Afterward, $5 \mathrm{~mL}$ of the supernatant was mixed well with $5 \mathrm{~mL}$ of hydrochloric acid $(7: 93, v / v)$. The mixture was placed in a water bath at $90{ }^{\circ} \mathrm{C}$ for $30 \mathrm{~min}$, followed by ethyl acetate extraction 3 times. Then, $0.4 \mathrm{~mL}$ of the extract was placed in a $10 \mathrm{~mL}$ colorimetric tube and dried; after the addition of $0.4 \mathrm{~mL}$ of vanillin-glacial acetic acid $(5: 95, v / v)$ and $1.6 \mathrm{~mL}$ of perchloric acid, the sample was placed in a water bath at $60^{\circ} \mathrm{C}$ for $15 \mathrm{~min}$ and mixed with $8 \mathrm{ml}$ of glacial acetic acid. Finally, absorbance was measured at $545 \mathrm{~nm}$, and total saponin levels were calculated based on a standard curve generated with ursolic acid.

\subsection{Determination of Polysaccharide Amounts}

Polysaccharides were extracted from I. asprella and quantified as described previously [11]. One gram of I. asprella powder was diluted in $50 \mathrm{~mL}$ of water, followed by ultrasonication for $60 \mathrm{~min}$; the sample was diluted to $200 \mathrm{~mL}$ after filtration. Then, $2 \mathrm{~mL}$ of the solution was mixed with $1 \mathrm{~mL}$ phenol and $5 \mathrm{~mL}$ vitriol, and placed $10 \mathrm{~min}$ before incubation in a water bath at $40{ }^{\circ} \mathrm{C}$ for $15 \mathrm{~min}$. Absorbance was measured at $490 \mathrm{~nm}$ on an ultraviolet spectrophotometer. The polysaccharide content was calculated based on a standard curve generated with glucose. 


\subsection{Statistical Analysis}

Stat-Ease software (Design-Expert 8.0.6.1 version, Stat-Ease Corporation, Minneapolis, MN, USA, 2015) was used for the regression analysis of the data, and to plot the response surface graphs. The variability and accuracy of the model were determined according to the regression coefficient $\left(R^{2}\right)$ and lack of fit, respectively. Analysis of variance (ANOVA) was performed and the values were considered significant when $p<0.05$. The response surface and contour plots of the predicted responses of the model were used to assess interactions between the significant factors. Additionally, numerical optimization was carried out by performing three-dimensional response surface analysis of the independent and dependent variables.

Author Contributions: Conceptualization and Methodology, H.X. and X.L.; Software, C.L. and X.L.; Investigation, W.W. and T.L; Writing-Original Draft Preparation, X.L.; Writing-Review \& Editing, H.X. and X.L.; Funding Acquisition, R.Z. and W.C.; Experimental Materials, Z.H. and J.X. All the authors have read and approved the final manuscript.

Funding: This research was supported by the Project Based Personnel Exchange Program between the China Scholarship Council and the German Academic Exchange Service, and the Science and Technology Project of Guangdong Province (Grant No.2015A030401083), and Guangdong Provincial Education Department Focuses on Improving Platform Construction Projects, Key Laboratory of Chinese Medicinal Resource from Lingnan, Ministry of Education (Grant No.2014KTSPT016).

Acknowledgments: We appreciate Runsheng Zheng and Xiaoyu Ji for technical support with LC-MS/MS analysis.

Conflicts of Interest: The authors declare no conflict of interest.

\section{References}

1. Biselli, S. Analytische Methoden für die Kontrolle von Lebens- und Futtermitteln auf Mykotoxine. J. Verbrauch. Lebensm. 2006, 1, 106-114. [CrossRef]

2. Aiko, V.; Mehta, A. Prevalence of toxigenic fungi in common medicinal herbs and spices in India. 3 Biotech 2016, 6, 159. [CrossRef] [PubMed]

3. Halt, M. Moulds and mycotoxins in herb tea and medicinal plants. Eur. J. Epidemiol. 1998, 3, $269-274$. [CrossRef]

4. Baoyu, Z. Research progress of sterigmatocystin. Pro. Vet. Med. 1997, 2, 18-21.

5. Yu, J.; Chang, P.K.; Ehrlich, K.C.; Cary, J.W.; Bhatnagar, D.; Cleveland, T.E.; Payne, G.A.; Linz, J.E.; Woloshuk, C.P.; Bennett, J.W. Clustered Pathway Genes in Aflatoxin Biosynthesis. Appl. Environ. Microbiol. 2004, 70, 1253-1262. [CrossRef] [PubMed]

6. Versİ ilovskis, A.; De Saeger, S. Sterigmatocystin: Occurrence in foodstuffs and analytical methods-An overview. Mol. Nut. Food Res. 2010, 54, 136-147. [CrossRef] [PubMed]

7. Vesonder, R.F.; Horn, B.W. Sterigmatocystin in dairy cattle feed contaminated with Aspergillus versicolor. Appl. Environ. Microbiol. 1985, 49, 234-235. [PubMed]

8. Cai, W. Study on the cause of "yellow stain" in Ningxia sheep. J. Nanjing Agric. Univ. 1991, 14, 70-76.

9. Zheng, R.; Xu, H.; Wang, W.; Zhan, R.; Chen, W. Simultaneous determination of aflatoxin B1, B2, G1, G2, ochratoxin A, and sterigmatocystin in traditional Chinese medicines by LC-MS-MS. Anal. Bioanal. Chem. 2014, 406, 3031-3039. [CrossRef] [PubMed]

10. Du, B.Z.; Yang, X.Y.; Feng, X.; Yin, X.; Zhang, H.X.; Zhao, F.; Gao, Z.P.; Tu, P.F.; Chai, X.Y. A phytochemical and pharmacological advance on Ilex asprella. CJCMM 2017, 1.

11. Wei, Y. Determination of polysaccharides in Ficus carica by phenol sulfuric acid method. Food Sci. Tech. 2009, 34, 256-262.

12. Bellemain, E.; Carlsen, T.; Brochmann, C.; Coissac, E.; Taberlet, P.; Kauserud, H. ITS as an environmental DNA barcode for fungi: An in silico approach reveals potential PCR biases. BMC Microbiol. 2010, 10, 189. [CrossRef] [PubMed]

13. Dong, F.; Qiu, J.; Xu, J.; Yu, M.; Wang, S.; Sun, Y.; Zhang, G.; Shi, J. Effect of environmental factors on Fusarium population and associated trichothecenes in wheat grain grown in Jiangsu province, China. Int. J. Food Microbiol. 2016, 230, 58-63. [CrossRef] [PubMed] 
14. West, J.S.; Holdgate, S.; Townsend, J.A.; Edwards, S.G.; Jennings, P.; Fitt, B.D.L. Impacts of changing climate and agronomic factors on fusarium ear blight of wheat in the UK. Fungal Ecol. 2012, 5, 53-61. [CrossRef]

15. Singh, P.; Shera, S.S.; Banik, J.; Banik, R.M. Optimization of cultural conditions using response surface methodology versus artificial neural network and modeling of L-glutaminase production by Bacillus cereus MTCC 1305. Bioresource Technol. 2013, 137, 261-269. [CrossRef] [PubMed]

16. Wu, L.; Qiu, L.; Zhang, H.; Sun, J.; Hu, X.; Wang, B. Optimization for the Production of Deoxynivalenoland Zearalenone by Fusarium graminearum Using Response Surface Methodology. Toxins 2017, 9, 57. [CrossRef] [PubMed]

17. Muralidhar, R. Racemic resolution of RS-baclofen using lipase from Candida cylindracea. Mededelingen 2001, 66, 227-232. [PubMed]

18. Kontogiannopoulos, K.N.; Patsios, S.I.; Karabelas, A.J. Tartaric acid recovery from winery lees using cation exchange resin: Optimization by Response Surface Methodology. Sep. Pur. Technol. 2016, 165, 32-41. [CrossRef]

19. Sahoo, C.; Gupta, A.K. Optimization of photocatalytic degradation of methyl blue using silver ion doped titanium dioxide by combination of experimental design and response surface approach. J. Hazard. Mater. 2012, 302-310. [CrossRef] [PubMed]

20. Abramson, D. Mycotoxin formation in hulless barley during granary storage at 15 and $19 \%$ moisture content. J. Stored Prod. Res. 1999, 35, 297-305. [CrossRef]

21. Tian, H. Survey and analysis on sterigmatocystin contaminated in grains in China. J. Hygiene Res. 2004, 33, 606-608.

22. Müller, P.; Basedow, T. Aflatoxin contamination of pods of Indian Cassia senna L. (Caesalpinaceae) before harvest, during drying and in storage: Reasons and possible methods of reduction. J. Stored Prod. Res. 2007, 43, 323-329. [CrossRef]

(C) 2018 by the authors. Licensee MDPI, Basel, Switzerland. This article is an open access article distributed under the terms and conditions of the Creative Commons Attribution (CC BY) license (http:// creativecommons.org/licenses/by/4.0/). 